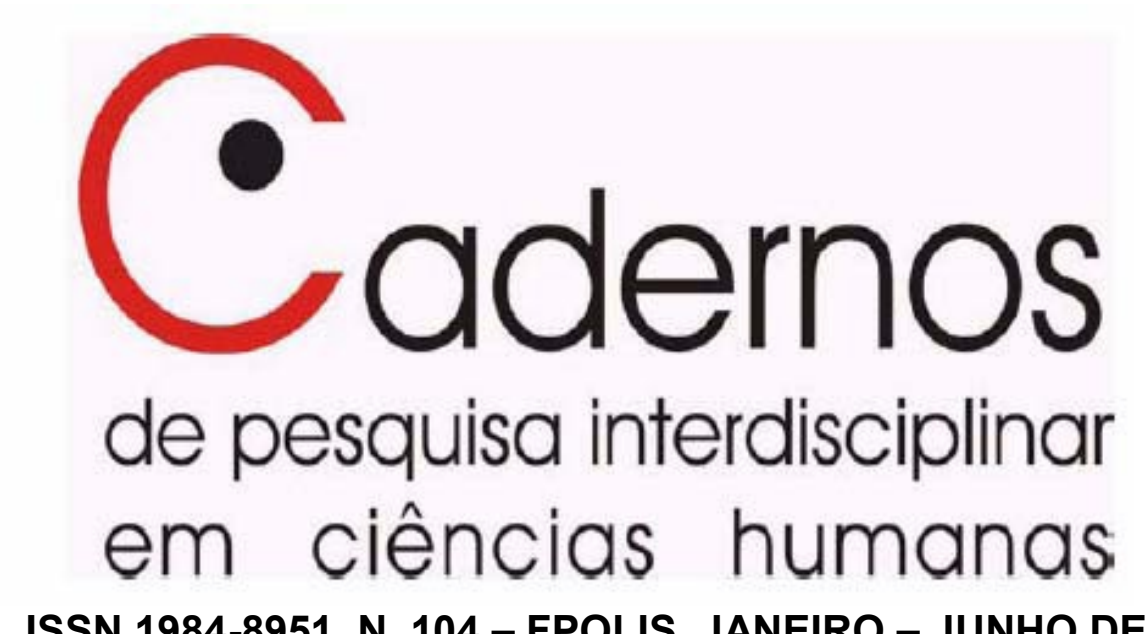

ISSN 1984-8951, N. 104 - FPOLIS, JANEIRO - JUNHO DE 2013

\title{
Editora
}

Myriam Raquel Mitjavila, UFSC - Florianópolis - SC, Brasil

\section{Conselho Editorial}

Carmen Rial, UFSC - Florianópolis - SC, Brasil

Cristiana Bastos, Universidade de Lisboa, Portugal

Francisco Javier García Marco, Universidad de Zaragoza, Espanha

Gabriel Zárate Guerrero, Universidad de Guadalajara - México, México

Hector Ricardo Leis, Universidade Federal de Santa Catarina - UFSC, Brasil

lluska Maria da Silva Coutinho, Universidade Federal de Juiz de Fora - UFJF, Brasil

Isis Fernandes Pinto, Universidade Livre de Berlim, Alemanha

James Naylor Green, Brown University, Estados Unidos da América do Norte

Julia Silvia Guivant, Universidade Federal de Santa Catarina - UFSC, Brasil José Filipe Pinheiro Chagas Verde, Instituto Superior de Ciências do Trabalho e da Empresa - ISCTE, Portugal

Luiz Eduardo Prado de Oliveira, Université de Bretagne Occidentale.

Margarete Axt, Universidade Federal do Rio Grande do Sul - UFRGS, Brasil María del Carmen Espinosa Gómez, Universidad de Guadalajara - México, México

María Dolores Pérez Murillo, Universidad de Cádiz, Espanha

María Lucrecia Rovaletti, Universidad de Buenos Aires - UBA

Maria Virginia Filomena Cremasco, Universidade Federal do Paraná - UFPR, Brasil

Marta Braga de Matos, Universidade Fernando Pessoa, Portugal

Massimo Canevacci, Università degli Studi di Roma "la Sapienza", Itália

Miriam Pillar Grossi, UFSC -Florianópolis - SC, Brasil

Raquel Wiggers, Universidade Federal do Amazonas - UFAM, Brasil 
Rosângela Miranda Cherem, Universidade do Estado de Santa Catarina, Brasil Sandra Makowiecky, Universidade do Estado de Santa Catarina - UDESC, Brasil Sandra Regina Ramalho e Oliveira, Universidade do Estado de Santa Catarina UDESC, Brasil

Selvino José Assmann, UFSC -Florianópolis - SC, Brasil

Valquíria Aparecida Passos Kneipp, Universidade Federal do Rio Grande do Norte UFRN/Universidade de Fortaleza - UNIFOR, Brasil

\section{Editora Associada}

Fernanda Rebelo, Universidade Federal de Santa Catarina - UFSC, Brasil

\section{Editoras Executivas}

Priscilla Mathes, Universidade Federal de Santa Catarina - UFSC, Brasil Ethel Scliar Cabral, Universidade Federal de Santa Catarina - UFSC, Brasil

\section{Editores Assistentes}

Paulo S. R. de Paula, Universidade Federal de Santa Catarina - UFSC, Brasil Maria Fernanda Vásquez, Universidad de Antioquia, Colômbia

\section{Bolsistas}

Diogo Zanoni Casagrande, Universidade Federal de Santa Catarina - UFSC, Brasil Fernanda Maria Alves Lourenço, Universidade Federal de Santa Catarina - UFSC,

\section{Brasil}

\section{Estagiário}

Mauricio Álvarez Valdés, Universidad Católica Silva Henríquez, Chile

\begin{tabular}{|l} 
(Catalogação na fonte pela DECTI da Biblioteca da UFSC) Cadernos de pesquisa \\
interdisciplinar em ciências humanas. \\
Universidade Federal de Santa Catarina. Centro de Filosofia e Ciências Humanas. \\
Programa de Pós-graduação Interdisciplinar em Ciências Humanas. - V. 14, n.104 (2013) - \\
Florianópolis: Universidade Federal de Santa Catarina, 2013 - \\
v.; $28 \mathrm{~cm}$ \\
Semestral \\
Resumo em português e inglês. A partir de maio de 2008, disponível no portal de \\
periódicos UFSC \\
em: http://www.periodicos.ufsc.br \\
ISSN 1984-8951 \\
E-ISSN \\
1. Abordagem interdisciplinar do conhecimento. 2. Ciência e humanidades. 3. Periódico I. \\
Universidade Federal de Santa Catarina.
\end{tabular}

